\title{
A comparison of the local structure in ball-milled and hand ground skutterudite samples using EXAFS
}

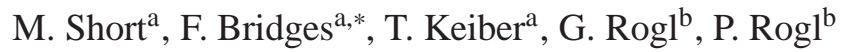 \\ ${ }^{a}$ Physics Department, University of California, Santa Cruz, California 95064, USA \\ ${ }^{b}$ Institute of Physical Chemistry, Christian Doppler Laboratory for Thermoelectrics, Währingerstraße 14, A-1090 Wien, Austria
}

\begin{abstract}
Skutterudites are considered to be good thermoelectrics with high figures of merit, ZT. After synthesis, electrodes are created by grinding and hot pressing the resulting powder. Materials such as $\mathrm{NdFe}_{4} \mathrm{Sb}_{12}$ exhibit a significantly greater figure of merit, ZT, (about $43 \%$ ) when it is ball milled to produce fine powders (inital powder $160 \mathrm{~nm}$; after hot pressing $330 \mathrm{~nm}$ ). This enhancement is typically attributed to the reduced particle size, which in turn decreases the mean free path of phonons, and consequently decreases the thermal conductivity. This work aims to investigate whether there is any damage to the crystal structure in the particles formed by ball milling, which could also affect its thermal conductivity. Using a temperature dependent, Extended X-ray Absorption Fine Structure (EXAFS) analysis of a hand ground and ball milled sample of the skutterudite $\mathrm{Nd}_{\mathrm{y}} \mathrm{Fe}_{4} \mathrm{Sb}_{12}$, we have determined that ball milling causes no significant damage to the local structure around any site. Consequently further improvements in $Z T$ may be possible with smaller particles.
\end{abstract}

Keywords: Extended x-ray absorption fine structure, thermoelectric proprieties, mechanical alloying and milling, microstructure, thermoelectric power generation

\section{Introduction}

Because of their unique structure and behavior, skutterudites present us with tantalizing potential applications in thermoelectrics. They take on the cubic space group $\operatorname{Im} \overline{3}$ and the simplest compounds have the chemical formula $M X_{3}$ (where, for example, $M=\mathrm{Co}$ and $X=\mathrm{Sb}$ ). The unit cell is shown in Fig. 1 from two different perspectives, and consists of 8 individual small cubic cells, 6 of which contain a ring of $X$ atoms and two are empty for $\mathrm{CoSb}_{3}$. However, the other two sub-cubes are usually filled with a rare earth metal giving it a relatively weakly bonded "rattler" atom [1]. These are termed filled skutterudites with the chemical formula[2], $R_{\mathrm{y}} M_{4} X_{12}$, where $R$ is a rare earth metal, $\mathrm{y}$ is the rattler filling fraction, $M$ is a transition metal including $\mathrm{Fe}, \mathrm{Ru}$ and $\mathrm{Os}$ and $X$ is a pnictogen including $\mathrm{P}, \mathrm{As}$ and $\mathrm{Sb}$. Here we only consider $M=\mathrm{Fe}$, and $X=\mathrm{Sb}$. The unit cell can also be viewed as tilted $\mathrm{Sb}$-octahedra about each $\mathrm{Fe}$ site - Fig. 1:bottom. Note that the shortest Sb-Sb distances (2.94 and $2.99 \AA$ ) are in the ring structure which connects four Sb-octahedra.

Introducing rattler atoms in this structure leads to a significantly reduced thermal conductivity, $\kappa,[3]$ although the actual mechanism[4, 5] for phonon scattering is still under debate. This in turn significantly increases the material's figure of merit, ZT, (a measure of thermoelectric quality), where

${ }^{*}$ Corresponding author

$$
Z T=\frac{T S^{2} \sigma_{e}}{\kappa},
$$

(C) 2015. This manuscript version is made available under the Elsevier user license http:/www.elsevier.com/open-access/userlicense/1.0/ 

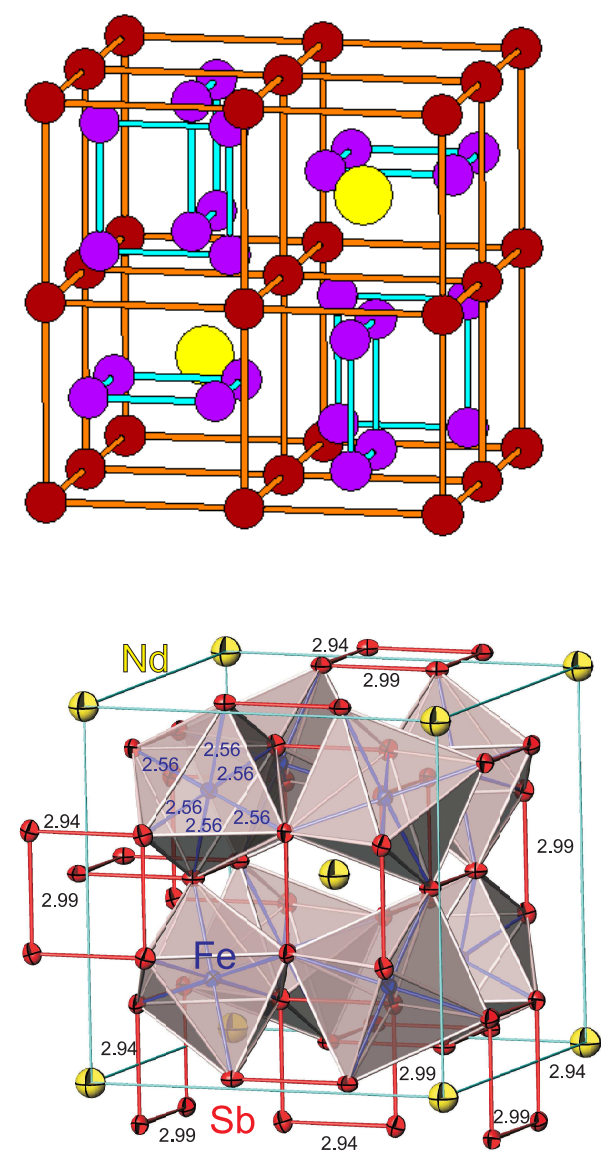

Figure 1: The filled skutterudite structure. The top figure emphasizes the ring and rattler locations for this case, red is a transition metal, blue is a pnictogen, and yellow is a rare earth metal. The lower figure features octahedra of $\mathrm{Sb}$ around the $\mathrm{Fe}$ atoms and shows how they are tilted. The shortest $\mathrm{Sb}-\mathrm{Sb}$ distances (2.94 and $2.99 \AA$ ) are between four octahedra and form the ring structures.

$T$ is temperature, $S$ is the Seebeck Coefficient, and $\sigma_{e}$ is the electrical conductivity [6].

A further means of reducing $\kappa$ is to decrease the phonon mean free path by using smaller particle sizes, produced via ball milling. Note that because electron mean free paths (which control the electrical conductivity) are already much shorter, the reduced particle size has little effect on $\sigma_{e}$. Such an approach has been successfully applied for the filled skutterudite $\mathrm{Nd}_{0.86} \mathrm{Fe}_{4} \mathrm{Sb}_{12}$; after ball milling to produce small particles of order $160 \mathrm{~nm}$ and hot pressing which roughly doubled the size, ZT increased by $43 \%$ at $800 \mathrm{~K}$ (from 0.74 to 1.06 )[7].
However, it is also possible that the ball milling process damages the local structure and bonding characteristics of the skutterudite, and in some cases can lead to amorphization[8,9] which would also affect the mean free path of the phonons. If the resulting sample were a mix of small crystallites and highly disordered/amorphous material, $\mathrm{x}$ ray diffraction would see the crystallites but miss the amorphous component. In contrast, EXAFS (Extended X-ray Absorption Fine Structure) measurements are sensitive to all atoms in the sample and if there were a significant fraction of disordered material, the amplitudes of the EXAFS peaks at low $\mathrm{T}$ would be smaller than expected. In this paper we will use temperature dependent EXAFS measurements to compare the disorder and bond strengths in $\mathrm{Nd}_{\mathrm{y}} \mathrm{Fe}_{4} \mathrm{Sb}_{12}$ for all three sites $(\mathrm{Nd}, \mathrm{Fe}$ and $\mathrm{Sb}$ ), between a hand ground ( $\mathrm{HG}$ ) and a ball milled (BM) sample.

\section{Experimental Details}

The sample materials were prepared via a master alloy, using an annealing-reaction-melting technique followed by hand grinding or ball milling and hot pressing as described in detail in Ref. [7] The rare earth used was the less expensive metal, Didymium - a mixture of $\sim 95 \% \mathrm{Nd}$ and $5 \% \mathrm{Pr}$. The BM sample was ground in a rotating drum containing hard metallic spheres to obtain particles initially $\sim 166 \mathrm{~nm}$ in size. In both cases the resulting powders were hot pressed (56 MPA uniaxial pressure, $\mathrm{T}=700{ }^{\circ} \mathrm{C}$, Ar gas, for $30 \mathrm{~min}$.) which increased the particle size to $\sim 330 \mathrm{~nm}$. This is the same material for which the improved ZT $=1.1$ was reported[7].

There are several different particle size designations and different means of estimating them. In the hot pressed samples, individual grains can be observed in SEM pictures - but within each grain there are small crystallites - small pieces of single crystal that scatter x-rays coherently. During hot pressing some nanoparticles grow together making somewhat larger crystallites. Also for powders some individual particles may contain multiple crystallites. A recent paper[10] discusses these 
various size measurements and some general details are included in the supplement along with SEM images for these samples.

The grain size is less well known and was determined from broken surfaces in the SEM images see supplement. These sizes were 0.1-0.4 $\mu \mathrm{m}$ for the ball-milled and 1-4.5 $\mu \mathrm{m}$ for the hand ground samples.

For the EXAFS samples, the hot pressed material was re-ground and passed through a sieve. These powders were brushed onto scotch tape which was then folded over to make a double layer; the resulting particle size was $\leq 5 \mu \mathrm{m}$. Several of these double layers were stacked on the sample holder to obtain the desired step height in the absorption data for a given edge. X-ray absorption data were collected at the Stanford Synchrotron Radiation Lightsource (SSRL) on beamline 4-1 for both $\mathrm{Fe}$ and $\mathrm{Sb} \mathrm{K}$ edges and beamline 7-3 for the Nd $\mathrm{L}_{\mathrm{III}}$ edge. Both beamlines used Si 220 monochromator crystals, detuned to $50 \%$ to minimize harmonics.

\section{Step Subtractions}

Most of the data reduction followed standard procedures and was carried out using the RSXAP package[11]. However, for these samples there is a slight interference from other edges and these edge steps had to be removed in the data processing. First, $\mathrm{Nd}$ came in the form of Didymium and therefore had a slight concentration $(\sim 5 \%)$ of $\mathrm{Pr}$. Since the Nd data were collected at the $\mathrm{L}_{\text {III }}$ edge at $6.21 \mathrm{keV}$, the Pr $\mathrm{L}_{\mathrm{II}}$ edge at $6.44 \mathrm{keV}$ was visible. To remove this, a function $f(E)=s(E)+\sum_{n} g_{n}(E)$ was fitted to the Pr $\mathrm{L}_{\mathrm{II}}$ edge from a separate energy space data file focusing on this edge. This consisted of a step function, $s(E)$, and a set of Gaussians, $g_{n}(E)$ where,

$$
\begin{gathered}
s(E)=\frac{A}{\pi}\left(\arctan \left(\frac{E-E_{0}}{\Delta}\right)+\frac{\pi}{2}\right) \\
g_{n}(E)=A_{n} \sqrt{\frac{1}{2 \pi \sigma_{n}^{2}} e^{-\frac{\left(E-E_{0_{n}}\right)^{2}}{2 \sigma_{n}^{2}}}},
\end{gathered}
$$
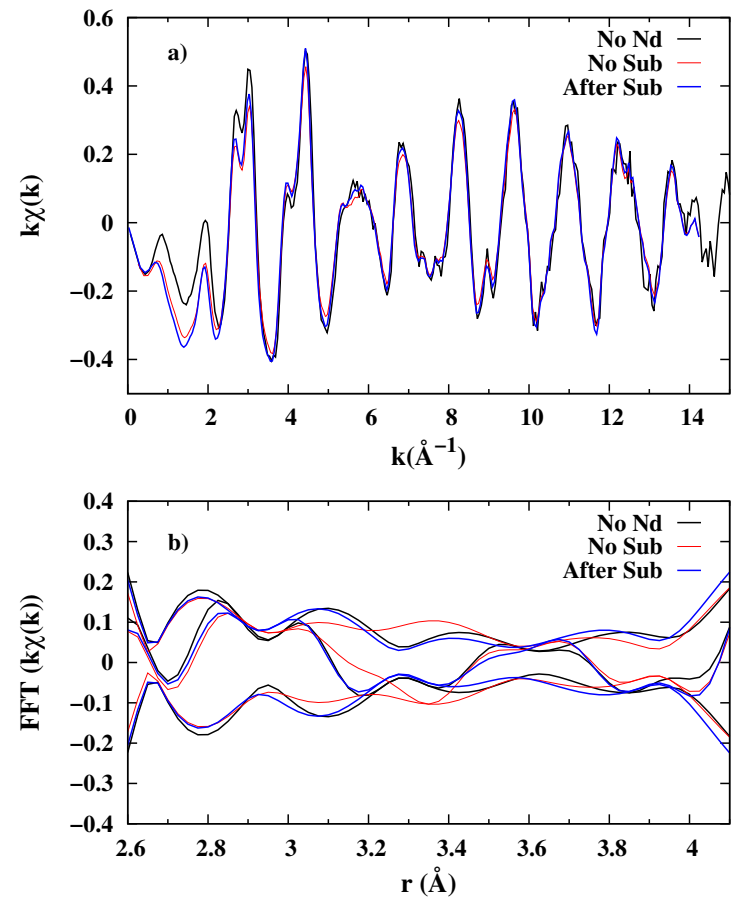

Figure 2: Comparison between $\mathrm{Fe} \mathrm{K}$ edge data from $\mathrm{CeFe}_{4} \mathrm{Sb}_{12}$ (No Nd) with the Fe K edge data from $\mathrm{NdFe}_{4} \mathrm{Sb}_{12}$, with and without the $\mathrm{Nd} \mathrm{L}_{\mathrm{I}}$ edge subtraction in: a) $k$-space and b) $r$-space. A tiny Nd-Sb peak from the $\mathrm{Nd} \mathrm{L}_{\mathrm{I}}$ can be seen in the unsubtracted $r$-space data between 3.0 and $3.5 \AA$, where there are no peaks in the $\mathrm{Fe} \mathrm{K}$ edge data. The corrected data plot is nearly identical to that for the $\mathrm{Fe} \mathrm{K}$ edge in $\mathrm{CeFe}_{4} \mathrm{Sb}_{12}$ which confirm the choice of $a$.

where $E$ is energy, $A$ is the amplitude of the step function, $\Delta$ is the width of the step, $E_{0}$ is the edge position for $\operatorname{Pr}, A_{n}$ are the amplitudes of the Gaussians, $\sigma_{n}^{2}$ are the "variances" or widths of each Gaussian and $E_{0_{n}}$ are the position of the Gaussian peaks. $f(E)$, in our case using three Gaussians, is then normalized to 1.0, and multiplied by the relative step height, $a$, of the $\operatorname{Pr} \mathrm{L}_{\mathrm{II}}$ step to the $\mathrm{Nd}$ $\mathrm{L}_{\mathrm{III}}$ edge, which was $\sim 0.05$. It was then subtracted from the normalized energy space data of $\mathrm{Nd}$ which were then renormalized. Since ball milling made use of manganese steel, a tiny Mn K edge at 6.54 $\mathrm{keV}$ was visible for one BM sample only. This was 
removed in the same manner as before but with only a step function fitted to the Mn edge visible in the $\mathrm{Nd}$ data near $6540 \mathrm{eV}$.

For the Fe K edge (7.112 keV) data a more complicated correction was needed to subtract the $\mathrm{Nd}$ $\mathrm{L}_{\mathrm{I}}$ edge $(7.126 \mathrm{keV})$ absorption, since it was difficult to distinguish the Nd EXAFS signal from that of the Fe. Therefore, a theoretical $k$-space file for the $\mathrm{Nd} \mathrm{L}_{\mathrm{I}}$ edge was created using the results from the fit to the Nd $\mathrm{L}_{\text {III }}$ data and the FEFF8 [12] program. The simulated $\mathrm{Nd} \mathrm{L}_{\mathrm{I}} k$-space file was then converted to energy space data with a unit step, multiplied by a coefficient $a$ and subtracted from our Fe edge. The coefficient $a$ was determined by multiplying the relative step heights for the two edges (0.555)[13] and the relative concentrations $\left(\frac{.85}{4}=0.213\right)$ of $\mathrm{Nd}$ and $\mathrm{Fe}$ in our sample giving an approximate value of 0.118 . The main effect of not including this correction is that the Fe $k$-space data are too small by roughly $10 \%$, and a tiny peak from the $\mathrm{Nd} \mathrm{L}_{\mathrm{I}}$ shows up between 3.0 and $3.6 \AA$ in the Fe $r$-space data, with a maximum at $3.35 \AA$. The lack of any remaining peak near $3.35 \AA$ in the subtracted data (Fig. 2) verifies that the magnitude of $a$ was appropriate. There is also a tiny remnant of the high $k$ region of the $\mathrm{Nd} \mathrm{L}_{\mathrm{II}}$ edge but it is very small in the pre-edge region and in the EXAFS region the contribution in $r$-space is at most a few percent. Also, these $\mathrm{L}_{\mathrm{II}}$ edge oscillations vary slowly with energy compared to the Fe EXAFS oscillations and are mostly removed in the spline fits of the background.

\section{Data and analysis}

After all the undesired edges were removed in energy space and the data were renormalized, the energy space data were transformed to $k$-space using the RSXAP package [14]. Figure 3 shows the low temperature results of this transformation where one can see the quality of data out to around 11 to $14 \AA^{-1}$. The data were then fast Fourier transformed (FFT) to r-space, as shown in Fig. 4, where the $k$-ranges were 5.5 to $13.5 \AA^{-1}$ for $\mathrm{Fe}, 3.8$ to 10.7 $\AA^{-1}$ for $\mathrm{Nd}$, and 3.8 to $13.7 \AA^{-1}$ for $\mathrm{Sb}$. One can see the large temperature dependence of the $\mathrm{Nd}_{\mathrm{III}}$

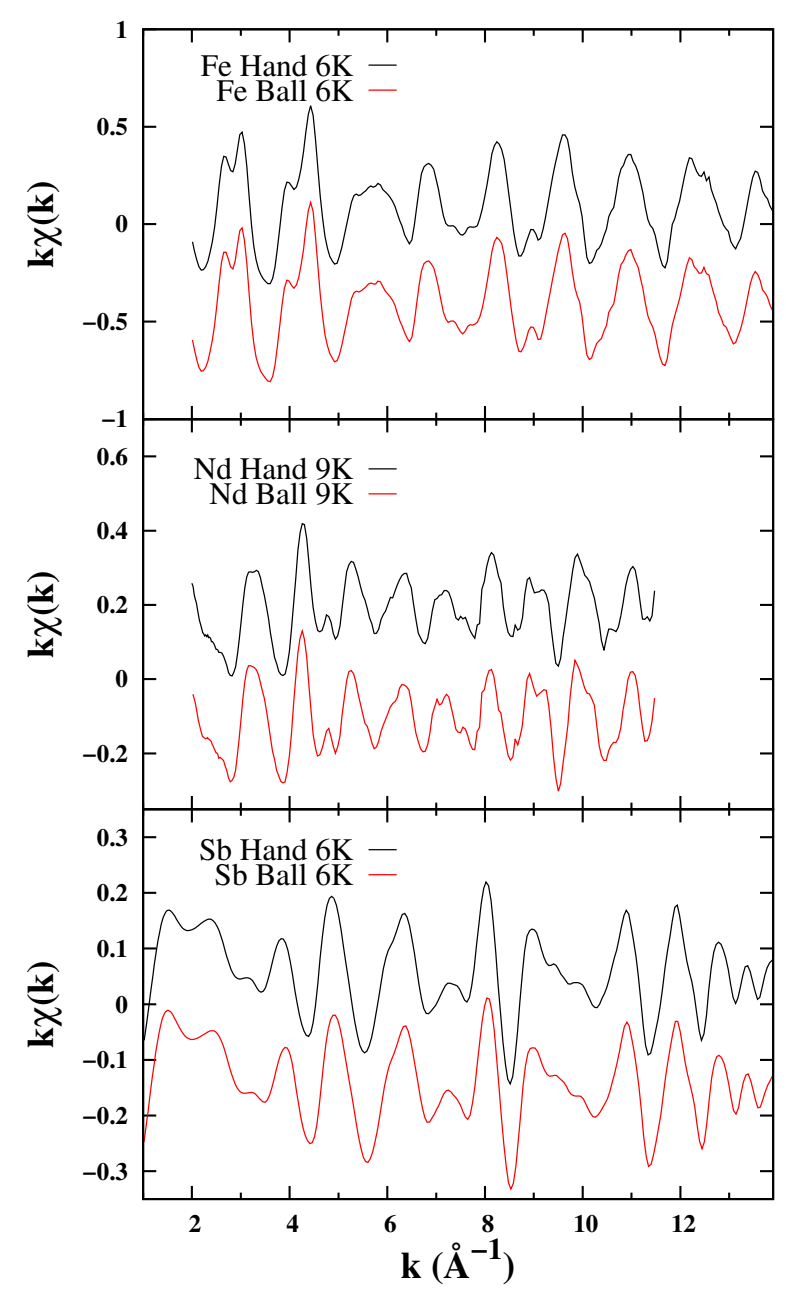

Figure 3: A comparison of the $k$-space data for the hand ground and ball milled samples at low temperature after contaminating edges (in the $\mathrm{Nd}$ and $\mathrm{Fe}$ data) were removed.

edge , dominated by the Nd-Sb pair, which shows the loose bonding of $\mathrm{Nd}$ and its rattler behavior. In addition, no significant change in the data is observable between the $\mathrm{HG}$ and $\mathrm{BM}$ samples for any edge in Fig. 4.

\subsection{Fits of the data}

The data for each edge/sample were fitted to theoretical $r$-space functions calculated using the ATOMS and FEFF8 [12] programs, assuming an $\operatorname{Im} \overline{3}$ structure with a lattice constant of a $=9.1355(3) \AA$ for the hand milled sample and $a=9.1361(2) \AA$ for the ball milled sample.[7] In the 


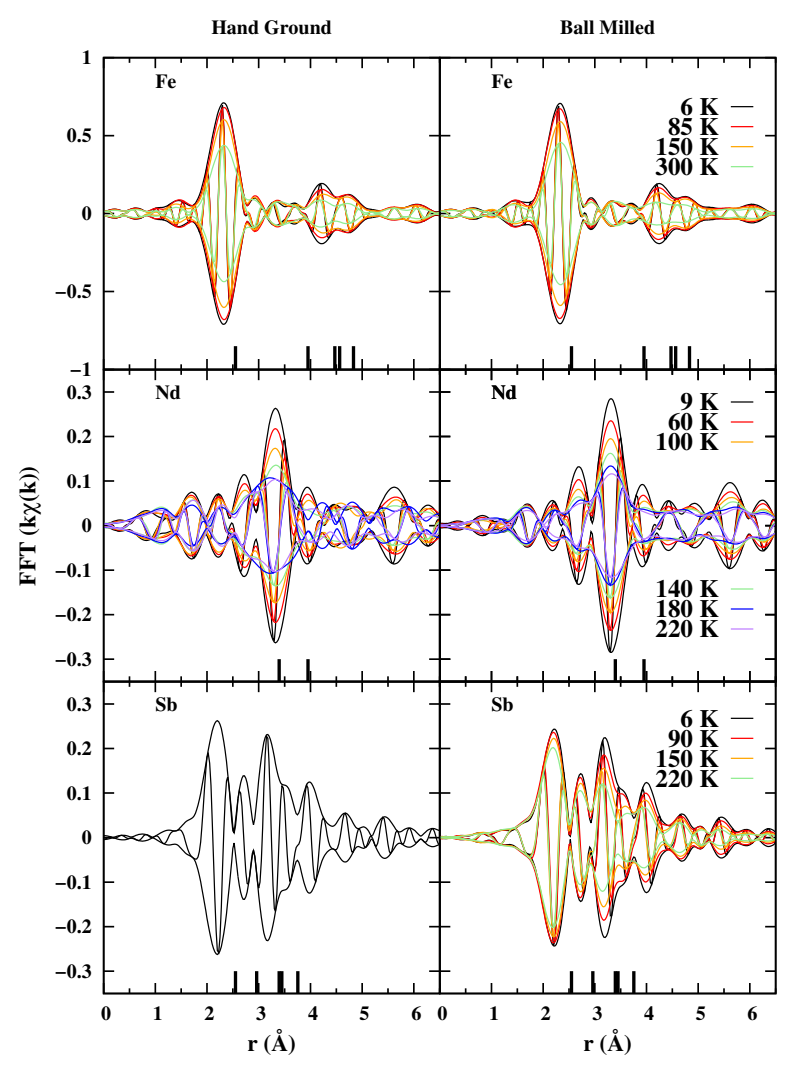

Figure 4: $r$-space data for the hand ground and ball milled samples as a function of temperature. The fast oscillations show the real part, $R$, of the FFT while the envelope is given by $\pm \sqrt{R^{2}+I^{2}}$ where $I$ is the imaginary part. Only the low $\mathrm{T}$ data are available for the $\mathrm{Sb}$ edge in the hand ground sample. The small vertical lines represent actual positions of various peaks; the EXAFS peaks are shifted to lower $r$ by a known phase factor. FT ranges: Fe, 5.5 to $13.5 \AA^{-1}$; Nd, 3.8 to 10.7 $\AA^{-1}$; Sb, 3.8 to $13.7 \AA^{-1}$.

initial fits, the ratio of the amplitudes was fixed to the ratio of the coordination numbers and only the first amplitude allowed to vary. Similarly the crystal structure was maintained by fixing the ratios of the different pair distances to the values obtained using the space group, with one distance allowed to vary. In order to get consistent results for $r$ and $\sigma^{2}$ as a function of $T$ however, the values for $\Delta E_{0}$ and $S_{0}^{2}$ first had to be determined from an average for the low temperature scans. Note that the peak amplitudes in the EXAFS equation are given by $\mathrm{A}_{i}=\mathrm{N}_{i} S_{0}^{2}$, where $\mathrm{N}_{i}$ is the coordination number for the $\mathrm{i}^{\text {th }}$ shell and $S_{0}^{2}$ is the amplitude reduction

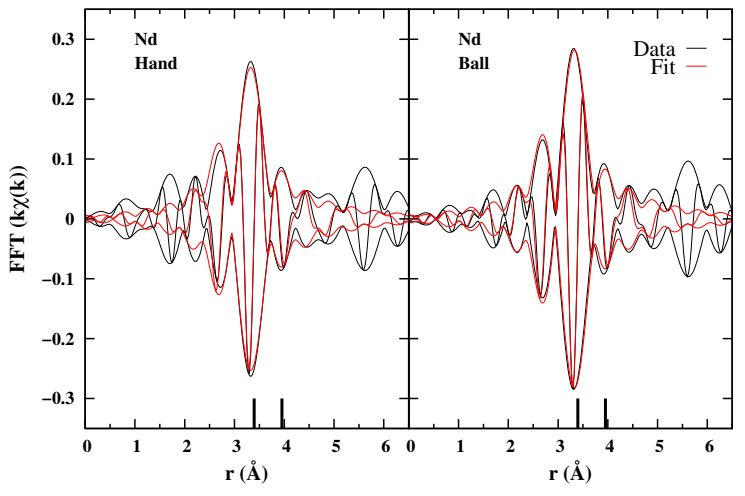

Figure 5: Two peak fit at the $\mathrm{Nd}_{\mathrm{IIII}}$ edge for $\mathrm{HG}$ and $\mathrm{BM}$ samples at $9 \mathrm{~K}$, from 2.5 to $4.0 \AA$. The two small vertical lines represent the actual pair distances: Nd-Sb at $3.396 \AA$ and NdFe at $3.953 \AA$.

factor from multi-electron excitations, with typical values between 0.7 and 1.0. The parameter, $\Delta E_{0}$, is the shift of the experimental edge position to correspond to $k=0$ in the theory. For isolated first peaks, such as the Fe-Sb peak, a one peak fit was used to determine $S_{0}^{2}$. Once $\Delta E_{0}$ and $S_{0}^{2}$ were determined, they were held fixed for fits as a function of $T$.

Fig. 5 shows an example of a fit of the $9 \mathrm{~K}, \mathrm{Nd}$ $r$-space data to two functions ( $\mathrm{Nd}-\mathrm{Sb}$ and $\mathrm{Nd}-\mathrm{Fe}$ pairs, with coordinations numbers of 12 and 8 ) over the range 2.5 to $4.0 \AA$; one overall lattice parameter and $\sigma$ 's for each peak were varied. Here, $S_{0}^{2}=0.75$ and the fit had 6 degrees of freedom.

The Fe K edge data were fit to a sum of five peaks as shown in Fig. 6 at $6 \mathrm{~K}$ using $S_{0}^{2}=0.78$; the parameters for the fifth peak are not reliable as overlapping further peaks are not included. The coordination numbers for the first four neighbors $(\mathrm{Sb}, \mathrm{Nd}, \mathrm{Sb}, \mathrm{Fe})$ are: 6, 2, 12, and 6. For the weak Fe-Nd peak, the value of $\sigma$ was held to that obtained from the Nd-Fe peak fit, extrapolated to match temperature points. This was done because the $\mathrm{Nd}$ peak in the Fe data is very small, having only two neighbors, while the fit to the $\mathrm{Nd} \mathrm{L}_{\text {III }}$ edge was a simple two peak fit, which fit very well as shown in Fig. 5. In constraining the parameters of the $\mathrm{Nd}$ peak in the Fe data, the effects on nearby peaks were minimized allowing a better quality fit. 


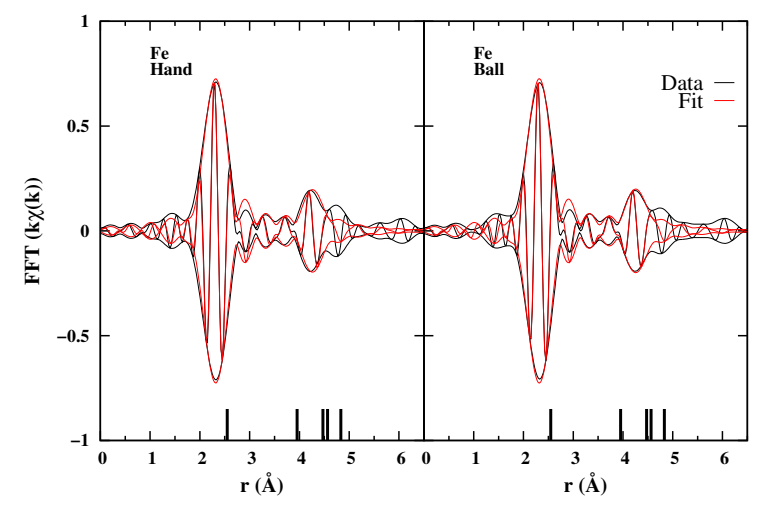

Figure 6: A five peak fit at the Fe $\mathrm{K}$ edge for the $\mathrm{HG}$ and $\mathrm{BM}$ samples at $6 \mathrm{~K}$, from 2.2 to $5.5 \AA$. The actual pair distances (vertical lines) are: $\mathrm{Fe}-\mathrm{Sb}$ at $2.549, \mathrm{Fe}-\mathrm{Nd}$ at $3.953, \mathrm{Fe}-\mathrm{Sb}$ at 4.473, and Fe-Fe at $4.565 \AA$, plus a multiscattering peak (Fe$\mathrm{Sb}-\mathrm{Fe}-\mathrm{Fe}$ ) with a net distance of $4.832 \AA$.

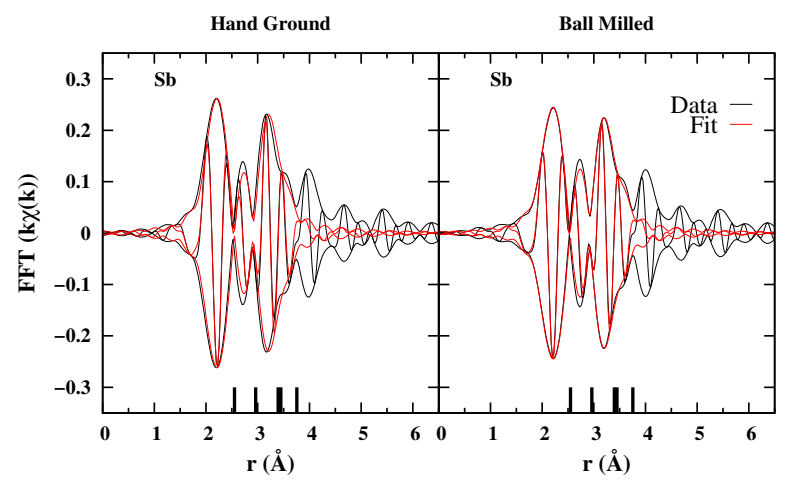

Figure 7: Five peak fit of $\mathrm{Sb} \mathrm{K}$ edge data for the $\mathrm{HG}$ and $\mathrm{BM}$ samples at $6 \mathrm{~K}$ from 1.9 to $3.75 \AA$. The small vertical lines represent the first five neighbors; Fe at $2.549, \mathrm{Sb}$ at $2.960, \mathrm{Nd}$ at 3.396, Sb at 3.448, and $\mathrm{Sb}$ at $3.756 \AA$.

A five peak fit to the $\mathrm{Sb}$ data at $6 \mathrm{~K}$ is shown in Fig. 7; $S_{0}^{2}=0.915$, and the coordination numbers are 2:2:1:4:4 for the first five neighbors ( $\mathrm{Fe}, \mathrm{Sb}$, $\mathrm{Nd}, \mathrm{Sb}, \mathrm{Sb}$ ). Again, $\sigma$ for the weak $\mathrm{Sb}-\mathrm{Nd}$ peak was held to the values obtained from the $\mathrm{Nd}-\mathrm{Sb}$ peak fit, extrapolated to match temperature points.

\subsection{Einstein/Debye Temperatures and Static Off- sets}

As can be seen in Fig. 4, the amplitudes of the peaks in $r$-space decrease as the temperature increases, showing an increase in $\sigma$. From fits similar to those shown in Fig. 5 to $7, \sigma^{2}$ was extracted for each pair as a function of $T$; these results are plotted for each sample, in Fig. 8. $\sigma^{2}(T)$ is a measure of the disorder for a given pair and is a sum of two components - a thermal vibration component, $\sigma_{v i b}^{2}$, and a static component, $\sigma_{\text {stat }}^{2}$; these effects add in quadrature to give $\sigma^{2}=\sigma_{\text {vib }}^{2}+\sigma_{\text {stat }}^{2}$. Depending on the behavior of the pair, one can fit a curve to the temperature dependence of $\sigma_{v i b}^{2}$ using either the correlated Debye or Einstein Models.

For the neighbors surrounding the $\mathrm{Nd}$ rattler atom, $\sigma^{2}$ for the first two atom pairs (Nd-Sb and $\mathrm{Nd}-\mathrm{Fe}$ ), displays a stronger temperature dependence in Fig. 8 than for any pairs in the $\mathrm{Fe}$ or $\mathrm{Sb}$ data. We can model the vibrations of the $\mathrm{Nd}-\mathrm{Sb}$ and Nd-Fe pairs using an Einstein Model as the rattler vibrates approximately as a simple harmonic oscillator inside a fairly stiff $\mathrm{Sb} / \mathrm{Fe}$ cage. For the Einstein model, $\sigma_{E}^{2}(T)$ is given by,

$$
\sigma_{E}^{2}(T)=\frac{\hbar^{2}}{2 M_{R} \mathrm{k}_{\mathrm{B}} \theta_{E}} \operatorname{coth}\left(\frac{\theta_{E}}{2 T}\right)+\sigma_{\text {stat }}^{2}
$$

where $M_{R}$ is the reduced mass of the pair being fit (in our case assuming a rigid cage, simply equal to the $\mathrm{Nd}$ mass), $\mathrm{k}_{\mathrm{B}}$ is the Boltzmann constant, $T$ is temperature and $\theta_{E}$ is the Einstein Temperature $\left(\theta_{E}=\hbar \omega_{E} / \mathrm{k}_{\mathrm{B}}\right)$.[15] In the case of pairs that are not connected to the rattler, the bonds are more rigid and vibration mode frequencies vary from 0 to $\omega_{D}$; in this case, the temperature dependence of $\sigma^{2}$ is better described using the correlated Debye model and the conventional reduced mass. The function describing this model is given by,

$$
\sigma^{2}(T)=\frac{3 \hbar}{2 M_{R}} \int_{0}^{\omega_{D}} \frac{\omega}{\omega_{D}^{3}} C_{i j} \operatorname{coth} \frac{\hbar \omega}{2 \mathrm{k}_{\mathrm{B}} T} d \omega+\sigma_{\text {stat }}^{2}
$$

where the correlation function $C_{i j}=1-$ $\frac{c}{\omega r_{i j}} \sin \left(\frac{\omega r_{i j}}{c}\right), c=\frac{\omega_{D}}{k_{D}}, k_{D}$ is the Debye wavenumber, $\omega_{D}$ is the Debye frequency and $r_{i j}$ is the distance to the $\mathrm{j}^{\text {th }}$ neighbor [16]. Here the correlated Debye temperature $\theta_{c D}=\hbar \omega_{D} / \mathrm{k}_{\mathrm{B}}$. 


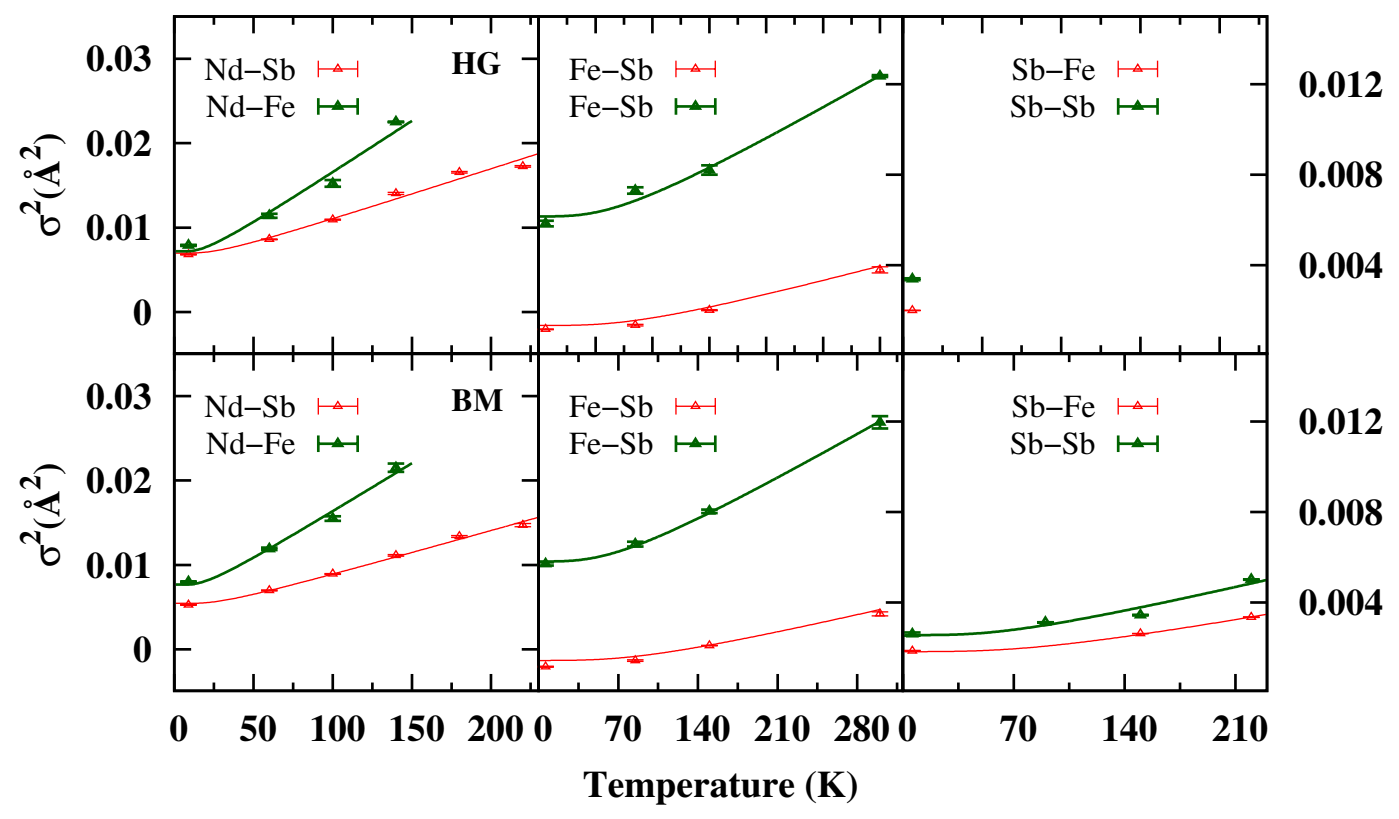

Figure 8: Plots of $\sigma^{2}(T)$ extracted from fits for each atom pair; upper row HG; lower row BM. The y-axis on the left refers to the $\mathrm{Nd}$ data while the one on the right refers to the $\mathrm{Fe}$ and $\mathrm{Sb}$ data. For the Fe data, the first and third neighbors are shown and for $\mathrm{Sb}$, the first and second neighbors are shown. The $\mathrm{Nd}$ neighbor for the $\mathrm{Fe}$ and $\mathrm{Sb}$ edges is already shown in the $\mathrm{Nd}$ data ( $\mathrm{Nd}-\mathrm{Sb}$ and $\mathrm{Nd}-\mathrm{Fe}$ ) and so is omitted in the plots for the $\mathrm{Fe}$ and $\mathrm{Sb}$ data.

If one considers the bond between the pair as a spring, the effective spring constant $K_{\mathrm{eff}}=M_{R} \omega^{2}=$ $M_{R}\left[\mathrm{k}_{\mathrm{B}} \theta / \hbar\right]^{2}$, will increase as $\omega_{E}$ or $\omega_{c D}$ increases. Therefore, $\theta_{E}$ and $\theta_{c D}$ (measured in Kelvin) are indicative of bond strength where lower values correlate with softer bonds.

The difference in $\theta_{E}$, see Table 1 , between the hand ground and ball milled samples is around 3$4 \%$ where variations between scans were around $5-6 \%$. Therefore, at the accuracy of our measurements there is no discernible difference between the samples. At high temperatures, we can see that the functions in Fig. 8 become very linear. This can be modeled at high $T$ by the equation $\sigma^{2}(T)=\mathrm{k}_{\mathrm{B}} T / K_{\text {eff }}$; consequently the inverse slope is a measure of the effective spring constant, $K_{\text {eff }}$. The high $\mathrm{T}$ slope was obtained by extrapolating the Einstein or correlated Debye fits to high T. A few effective spring constants are also tabulated in Ta- ble 1 .

Since for the hand ground sample we only have $6 \mathrm{~K}$ data at the Sb edge, no relationship between $\sigma^{2}$ and temperature could be determined, and results at the $\mathrm{Sb}$ edge for the HG sample are omitted in Table 1 . However in probing the local structure about $\mathrm{Sb}$ in this sample, the low $\mathrm{T}$ data are the most important. The values of $\sigma^{2}$ at $6 \mathrm{~K}$ for the HG sample are very similar to the values for the BM sample, indicating small values for any static disorder. Further, $\sigma^{2}(\mathrm{~T})$ for $\mathrm{Sb}-\mathrm{Fe}$ should be the same as $\sigma^{2}(\mathrm{~T})$ for $\mathrm{Fe}-\mathrm{Sb}$ in the Fe edge data (middle panels of Fig. 8), and the Fe-Sb curves for the HG and BM samples are nearly identical. Consequently, these results indicate that the temperature dependence of $\sigma^{2}$ at the $\mathrm{Sb} \mathrm{K}$ edge for the HG sample will be very similar to the results for the BM sample.

The unique behavior of the rattler site is clearly observed in Table 1 where other atom pairs have 


\begin{tabular}{|c|c|c|c|c|c|c|c|c|}
\cline { 4 - 9 } \multicolumn{4}{c|}{} & \multicolumn{4}{c|}{ Hand Ground } & \multicolumn{3}{c|}{ Ball Milled } \\
\hline Edge & Neighbor & $r(\AA)$ & $\sigma_{\text {stat }}^{2}\left(\AA^{2}\right)$ & $\theta(\mathrm{K})$ & $K_{\text {eff }}$ & $\sigma_{\text {stat }}^{2}\left(\AA^{2}\right)$ & $\theta(\mathrm{K})$ & $K_{\text {eff }}$ \\
\hline \multirow{2}{*}{$\mathrm{Nd}$} & $\mathrm{Sb}$ & 3.396 & 0.0047 & $75 \pm 4$ & 1.32 & 0.0031 & $78 \pm 1$ & 1.53 \\
\cline { 2 - 9 } & $\mathrm{Fe}$ & 3.953 & 0.0040 & $52 \pm 3$ & 0.94 & 0.0046 & $54.0 \pm 2$ & 0.96 \\
\hline \multirow{2}{*}{$\mathrm{Fe}$} & $\mathrm{Sb}$ & 2.549 & -0.0005 & $377 \pm 3$ & 6.41 & -0.0002 & $402 \pm 13$ & 7.17 \\
\cline { 2 - 9 } & $\mathrm{Sb}$ & 4.473 & 0.0032 & $319 \pm 9$ & 2.61 & 0.0028 & $319 \pm 3$ & 2.68 \\
\hline \multirow{2}{*}{$\mathrm{Sb}$} & $\mathrm{Fe}$ & 2.549 & - & - & - & 0.0001 & $386 \pm 8$ & 6.45 \\
\cline { 2 - 9 } & $\mathrm{Sb}$ & 2.960 & - & - & - & 0.0009 & $286 \pm 19$ & 4.85 \\
\hline
\end{tabular}

Table 1: Static offsets and Einstein or Debye Temperatures $\theta$, calculated from fitting equation 4 to the $\sigma^{2}$ plots for the Nd data, and equation 5 to the $\mathrm{Fe}$ and $\mathrm{Sb}$ data, in Fig. 8. Note that the errors provided for $\theta_{E}$ (Nd edge) or $\theta_{c D}$ (Fe and $\mathrm{Sb}$ edges) are relative errors from scatter in the values of $\sigma^{2}$, and do not account for systematic errors which are approximately $10 \%$. The systematic errors for $\sigma_{\text {stat }}^{2}$ are $\sim 0.0004 \AA^{2}$ for the Fe and Sb edge data and $\sim 0.001 \AA^{2}$ for the Nd edge data. Effective spring constants for the two closest neighbors are also tabulated (units eV/ $\AA^{2}$ ); they are calculated from $K_{\text {eff }}=\mathrm{k}_{\mathrm{B}} \Delta T / \Delta\left(\sigma^{2}\right)$ after extrapolating the fits of equations 4 or 5 to high temperatures. Estimated systematic errors $\sim 10 \%$. Corresponding values for the two samples agree except for the first $\mathrm{Fe}-\mathrm{Sb}$ pair in the ball milled sample, for which the spring constant is higher than expected and larger than for $\mathrm{Sb}-\mathrm{Fe}$ in same sample, which should be the same.

effective spring constants several times stiffer than for Nd-Sb or Nd-Fe. This table demonstrates that while there is some variance in our measurements, there is no significantly change in the behavior of any pair after ball milling.

\section{Discussion}

Our study on the structure and disorder of the filled skutterudite $\mathrm{Nd}_{y} \mathrm{Fe}_{4} \mathrm{Sb}_{12}$ focused primarily on the rattler site, as it is responsible for the nearly glass like thermal conductivity observed in these materials. A change in the stiffness of the bonding of $\mathrm{Nd}$ to the nearby $\mathrm{Sb}$ ring or $\mathrm{Fe}$ cage or a significant increase in $\sigma_{\text {stat }}^{2}$ for any pairs would directly affect the phonon scattering. The behavior of the Nd-Sb and Nd-Fe atom pairs were modeled using an Einstein model and values of $K_{\text {eff }}$ for these bonds estimated from the high $\mathrm{T}$ behavior. The low values for $K_{\text {eff }}$ indicate soft bonds - significantly softer than for pairs of atoms about the $\mathrm{Fe}$ or $\mathrm{Sb}$ sites. However, no significant difference was observed for $K_{\text {eff }}$ or for $\sigma_{\text {stat }}^{2}$ between the hand ground and ball milled samples for any of the atom pairs studied. The only static off-sets $\left(\sigma_{\text {stat }}^{2}\right)$ that are not essentially zero are those for the Nd-pairs, but even these are not large. For all Nd-Sb and Nd-Fe pairs static distortions are $\leq 0.07 \AA\left(\sqrt{\sigma_{\text {stat }}^{2}}\right)$; considering that there are a significant number of vacancies on the $\mathrm{Nd}$ sites, some $\mathrm{Sb}_{4}$ rings and/or $\mathrm{Nd}$ atoms may be slightly displaced if near a vacancy. Consequently this small value for $\sigma_{\text {stat }}$ does not indicate an off-center displacement.

The only direct comparison with other EXAFS studies is with Nitta et al.[17] who report EXAFS results at the $\mathrm{Nd} \mathrm{L}_{\mathrm{III}}$ for $\mathrm{NdFe}_{4} \mathrm{Sb}_{12}$, but not the other edges. Their value of $\theta_{E}$ for the Nd$\mathrm{Sb}$ pair is $\sim 197 \mathrm{~K}$, much much larger than our values - 75 and $78 \mathrm{~K}$; they also reported large $\theta_{E}$ for other compounds: $164 \mathrm{~K}$ for $\mathrm{CeFe}_{4} \mathrm{Sb}_{12}$ and $134 \mathrm{~K}$ for $\mathrm{PrFe}_{4} \mathrm{Sb}_{12}$. All these values are inconsistent with other measurements for rare earth rattlers in $R \mathrm{Fe}_{4} \mathrm{Sb}_{12}$ systems. Our earlier result for $\mathrm{Ce}-\mathrm{Sb}[18]$ found $\theta_{E}=86 \mathrm{~K}$, while inelastic neutron scattering[19] for $\mathrm{LaFe}_{4} \mathrm{Sb}_{12}$ observed an Einstein-like mode at $\sim 81 \mathrm{~K}(7 \mathrm{meV})$. These and our new results are reasonably consistent but about a factor of 2 smaller than Nitta et al.. In addition, another recent EXAFS study of phosphide skutterudites,[20] obtained $\theta_{E}$ values that ranged between 105 and $145 \mathrm{~K}$, depending on the rattler atom and the choice of the reduced mass. Our earlier value of $\theta_{E}$ for the Ce-P pair[18] in $\mathrm{CeFe}_{4} \mathrm{P}_{12}$ was $148 \mathrm{~K}$, reasonably consistent with these values. Because of the significantly smaller lattice parameters, $\theta_{E}$ is expected to be highest for the phosphides, and in systematic nuclear resonance inelastic scattering studies of the skutteru- 
dites $\mathrm{SmFe}_{4} X_{12}, X=\mathrm{P}, \mathrm{As}, \mathrm{Sb},[21]$ the phonon mode is indeed highest for $\mathrm{SmFe}_{4} \mathrm{P}_{12}(126 \mathrm{~K})$. Thus it is not clear why the values of Nitta et al. are so high for antimonides. In part, it is the use of the small simple reduced mass for the atom pair (we use the Nd mass while Mizumaki et al.[20] used both the rattler mass and a mass $\sim 78 \%$ of the rattler mass); however, it likely also arises from the smaller amplitude of the $\mathrm{Nd} \mathrm{L}_{\mathrm{III}}$ edge $k$-space data reported by Nitta et al.;117] our data (on a $k^{3} \chi(k)$ plot) is nearly a factor of two larger for $k$ near 10 $\AA^{-1}$. This smaller amplitude will also lead to an increased static distortion.

Based on these results we can say not only that the local structure of the skutterudite is essentially unchanged, but also that the vibrational properties of the rattler are unaffected by the ball milling process. Therefore, the increase in the figure of merit is primarily due to the increase in phonon scattering from the reduced particle size. Clearly it would be interesting to study the lower limit of nanoparticle sizes that could enhance the figure of merit while preserving the skutterudite structure and behavior of the rattler, without significantly reducing the mean free path for the charge carriers, thereby optimizing the thermoelectric performance. To achieve this, one would also need to investigate the range of particulate sizes created by different milling techniques so as to approach this limit without breaching it.

\section{Acknowledgments}

This work was supported under NSF grant DMR1005568. Use of the Stanford Synchrotron Radiation Lightsource, SLAC National Accelerator Laboratory, is supported by the U.S. Department of Energy, Office of Science, Office of Basic Energy Sciences under Contract No. DE-AC0276 SF00515.

\section{References}

[1] B. C. Sales, D. Mandrus, B. C. Chakoumakos, V. Keppens, J. R. Thompson, Filled skutterudite antimonides: Electron crystals and phonon glasses, Phys. Rev. B 56 (1997) 15081.
[2] W. Jeitschko, D. Braun, $\mathrm{LaFe}_{4} \mathrm{P}_{12}$ with filled $\mathrm{CoAs}_{3}-$ type structure and isotypic lanthanoid-transition metal polyphosphides, Acta. Crystallogr. B 33 (1977) 3401.

[3] D. T. Morelli, G. P. Meisner, Low temperature properties of the filled skutterudite $\mathrm{CeFe}_{4} \mathrm{Sb}_{12}$, J. Appl. Phys. 77 (1995) 3777-81.

[4] M. M. Koza, M. R. Johnson, R. Viennois, H. mutka, L. Girard, D. Ravot, Breakdown of phonon glass paradigm in La- and Ce-filled $\mathrm{Fe}_{4} \mathrm{Sb}_{12}$ skutterudites, $\mathrm{Na}$ ture Mater. 7 (2008) 805-10.

[5] W. Li, N. Mingo, Thermal conductivity of fully filled skutterudies: Roll of the filler, Phys. Rev. B. 89 (2014) 184384.

[6] G. D. Mahan, Good thermoelectrics, Vol. 51, Academic Press, New York, 1998, pp. 81-157.

[7] G. Rogl, A. Grytsiv, P. Rogl, E. Bauer, M. Zehetbauer, A new generation of p-type didymium skutterudites with high ZT, Intermetallics 19 (2011) 546-555.

[8] L. Zaluski, P. Tessier, D. Ryan, C. Doner, A. Zaluska, J. Strm-Olsen, M. Trudeau, R. Schulz, Amorphous and nanocrystalline Fe-Ti prepared by ball-milling, J. Mat. Res. 8 (1993) 3059-68.

[9] M. S. El-Eskandarany, Solid state nitrization reaction of amorphous tantalum aluminium nitride alloy powders: the role of amorphization by reactive ball milling, J. Alloys Cmpds. 203 (1994) 117-126.

[10] G. Rogl, A. Grytsiv, P. Rogl, E. Bauer, M. Hochenhofer, R. Anbalagan, R. C. Mallik, E. Schafler, Nanostructuring of p- and n-type skutterudites reaching figures of merit of approximately 1.3 and 1.6, respectively, Acta Mater 76 (2014) 434-448, http://dx.doi.org/10.1016/j.actamat.2014.05.051.

[11] See http://lise.lbl.gov/RSPAK.

[12] A. L. Ankudinov, B. Ravel, J. J. Rehr, S. D. Conradson, Real space multiple scattering calculation of xanes, Phys. Rev. B 58 (1998) 7565.

[13] W. H. McMaster, Compilation of X-ray Cross Sections, UCRL-50174 Sec. II, Rev. 1, Lawrence Radiation Laboratory, University of California, Berkeley, 1969.

[14] C. H. Booth, R-Space X-ray Absorption Package, 2010, http://lise.lbl.gov/RSXAP/ (2010).

[15] B. K. Teo, Springer-Verlag, New York, 1986, $\mathrm{dOI}=10.1007 / 978-3-642-50031-2$.

[16] D. Koningsberger, R. Prins (Eds.), Wiley, New York, 1988.

[17] K. Nitta, Y. Omori, T. Miyanaga, K. Takegahara, H. Sugawara, D. Kikuchi, H. Sato, Extended x-ray absorption fine structure thermal factor analysis of rattling in filled skutterudites $\mathrm{RT}_{4} \mathrm{Sb}_{12}$ (R: La, Ce, Pr, Nd, Sm; T: Fe, Ru, Os), J. Phys. Soc. Jpn. 82 (2013) 044801/1-5.

[18] D. Cao, F. Bridges, P. Chesler, S. Bushart, E. D. Bauer, M. B. Maple, Evidence for rattling of the filler atoms (1) in the filler skutterudites $\mathrm{LT}_{4} \mathrm{X}_{12}(\mathrm{~L}=\mathrm{Ce}, \mathrm{Eu}, \mathrm{Yb} ; \mathrm{T}=\mathrm{Fe}$, $\mathrm{Ru}$; $\mathrm{X}=\mathrm{P}, \mathrm{Sb}$ ) from exafs studies, Phys. Rev. B 70 (2004) 094109/1-11.

[19] J. L. Feldman, P. Dai, T. Enck, B. C. Sales, D. Mandrus, 
D. J. Singh, Lattice vibrations in $\mathrm{La}(\mathrm{Ce}) \mathrm{Fe}_{4} \mathrm{Sb}_{12}$ and $\mathrm{CoSb}_{3}$ : Inelastic neutron scattering and theory, Phys. Rev. B 73 (2006) 014306.

[20] M. Mizumaki, S. Tsutsui, T. Uruga, H. Tanida, D. Kikuchi, H. Sugawara, H. Sato, Rare earth dependence of einstein temperatures in filled skutterudite compounds $\mathrm{ReFe}_{4} \mathrm{P}_{12}(\mathrm{Re}=\mathrm{La}, \mathrm{Ce}, \mathrm{Pr}, \mathrm{Nd}, \mathrm{Sm}), \mathrm{J}$. Phys. Soc. Jpn. 80 (2013) 074603/1-6.

[21] S. Tsutsui, H. Kobayashi, , Y. Yoda, H. Sugawara, C. Sekine, T. Namiki, I. Shirotani, H. Sato, ${ }^{149} \mathrm{Sm}$ and ${ }^{57} \mathrm{Fe}$ nuclear resonant inelastic scattering of filled skutterudites $\mathrm{SmFe}_{4} \mathrm{X}_{12}$ (X: pnictogen), Hyperfine Interact 206 (2012) 67-70. 